\title{
Mathematical Foundations of Risk Analysis
}

\author{
Nozer Singpurwalla \\ The George Washington University, Emeritus Professor of Statistics, \\ The City University of Hong Kong, Adjunct Professor of Data Science
}

doi: https://doi.org/10.21467/abstracts.93.10

\begin{abstract}
ABSTRA C T
The mathematical theory of risk analysis rests on two pillars and one principle. The two pillars are probability and utility, and the principle is the maximization of expected utility (MEU). The latter is a consequence of the calculus of probability, particularly, the law of total probability. The law of total probability is germane here because of the argument that utilities are like probabilities and thus obey the laws of probability.

There are two general directions in which risk analysis can proceed; the traditional one wherein one faces nature, seen as a benevolent adversary, and adversarial wherein one faces an active adversary, like a terrorist. An approach to analyzing the first case is via decision trees and fault trees. In the latter case, an archetypal example of which is sequential drug testing, one needs to engage with what is known as a pre posterior analysis. Whereas classical decision trees take a finite number of stages, decision trees under pre posterior analysis can branch out indefinitely. This makes their analysis cumbersome, if not complicated.

The purpose of this expository talk is to articulate these and related matters outlining general principles as opposed to specific applications and scenarios. Audience interaction is expected and welcome.
\end{abstract}

(C) 2020 Copyright held by the author(s). Published by AIJR Publisher in "Abstracts of The Second Eurasian RISK-2020 Conference and Symposium" April 12- 19, 2020, Tbilisi, Georgia. Jointly organized by AMIR Technical Services LLC, Georgian Technical University, Institute of Geography (Kazakhstan) and Russian Institute of Petroleum Geology and Geophysics.

AijR DOI: $10.21467 /$ abstracts.93 